\title{
Reference Target Correction Based on Point Target SAR Simulation
}

\author{
Björn J. Döring, Philipp Looser, Matthias Jirousek, and Marco Schwerdt
}

\begin{abstract}
The backscattering from man-made point targets like passive corner reflectors and active transponders is often used as a radiometric calibration standard for synthetic aperture radar (SAR) calibration. As new systems emerge and the demand for more accurate systems increases, it becomes necessary to better understand the effects of real or imperfect targets on the radiometric calibration results. Therefore, a point target SAR simulator is presented which models the complete external radiometric calibration process. It incorporates a number of target properties like frequency response, transponder internal calibration strategies, noise, and interference signals, and it takes the instrument SAR mode settings into consideration. Thereby, the relevant target backscatter variation as observed in the processed SAR image with respect to an ideal or any other target can be determined.

The simulation results are relevant during the design process of a new target as well as during the actual calibration of a SAR system. Based on these point target simulations, correction coefficients can be stated for each target and SAR mode, therefore decreasing the remaining radiometric point target uncertainties. The quantitative examples in this paper show that these corrections can influence the absolute radiometric calibration by more than $1 \mathrm{~dB}$.
\end{abstract}

\section{INTRODUCTION}

A synthetic aperture radar (SAR) instrument maps the radar reflectivity of the earth's surface for a variety of remotesensing tasks. Depending on the application, a different degree of radiometric calibration is required. In the order of increasing complexity, this can be either (a) relative radiometric calibration within one scene, (b) relative radiometric calibration within all scenes of one mission, or (c) absolute radiometric calibration. A physical unit can only be attached to the measured reflectivity map after radiometric calibration. The first case (a) is sufficient, for instance, for visual feature extraction. Exemplary, the second type is a prerequisite for multi-temporal coverage, the derivation of repeat-pass interferometric images, or relatively calibrated polarimetric channels. On the other hand, absolute radiometric calibration is beneficial or required for

- Derivation of physical parameters like soil moisture from reflectivity measurements

- Automatic feature extraction and classification for instance for land usage maps

- Comparability and cross-calibration between different SAR instruments

It has to be noted that absolutely calibrated images are the most versatile, as they inherently include relative calibration. This

The authors are with the Microwaves and Radar Institute of the German Aerospace Center (DLR), Oberpfaffenhofen, Germany.

Manuscript received Month DD, 2011; revised Month DD, 2011. is the reason why SAR image products are usually delivered absolutely calibrated.

Absolute radiometric calibration is achieved by deploying a man-made reference point target (for instance an active transponder or a passive trihedral corner reflector) of known radar cross section (RCS) within an imaged scene [1]-[3]. Sometimes, also natural distributed targets like a patch of rainforest is used for this purpose [4], although the verification of the achievable accuracy for these targets is difficult. Once an image containing the reference target has been acquired, absolute radiometric calibration is accomplished by comparing the reflectivity of each image pixel (i.e. the pixel energy) with the reflectivity of the known target.

To the best of our knowledge, for the approach based on point targets ideal target properties have always been assumed. Distortions (especially for active transponders) caused by the actual point target transfer function, the introduction of spurious signals, or noise are some of the point-target deficiencies which have been neglected without fully understanding their effects on the reference RCS. Against the backdrop of a demand for increased absolute radiometric accuracies and the wish to build more accurate point targets, this approximate approach shall be reassessed. For instance, for the first civilian space-borne SAR system SEASAT (launched 1978) calibration was still a research topic [5], the German TerraSAR-X system (launched 2007) already exceeds the accuracy requirement of $0.9 \mathrm{~dB}$ (at a confidence level of $68 \%$ ) [2], whereas for the upcoming Sentinel-1 mission (launch planned for 2012) an overall absolute radiometric accuracy of $0.3 \mathrm{~dB}$ (at the same confidence level) is targeted [6]. Of course, the point target uncertainty is only one element in the overall SAR system error budget, and therefore its accuracy must be a fraction of the respective system accuracy.

To overcome the implicit uncertainties in assuming ideal point target properties and to gain a better understanding of the impact of point target deficiencies on its reflectivity, the application of a specialized point-target SAR simulator is proposed. This is particularly advantageous for active point targets (transponders). The adoption of such a simulator has advantages in two phases:

1) In the transponder design phase, the development engineer gains a practical tool with which RF component requirements (e.g. amplitude and phase response) can be specified with respect to the required overall transponder accuracy.

2) During the SAR system calibration, a target correction coefficient (TCC) can be derived for each target. This is specific to the actual instrument SAR mode settings 


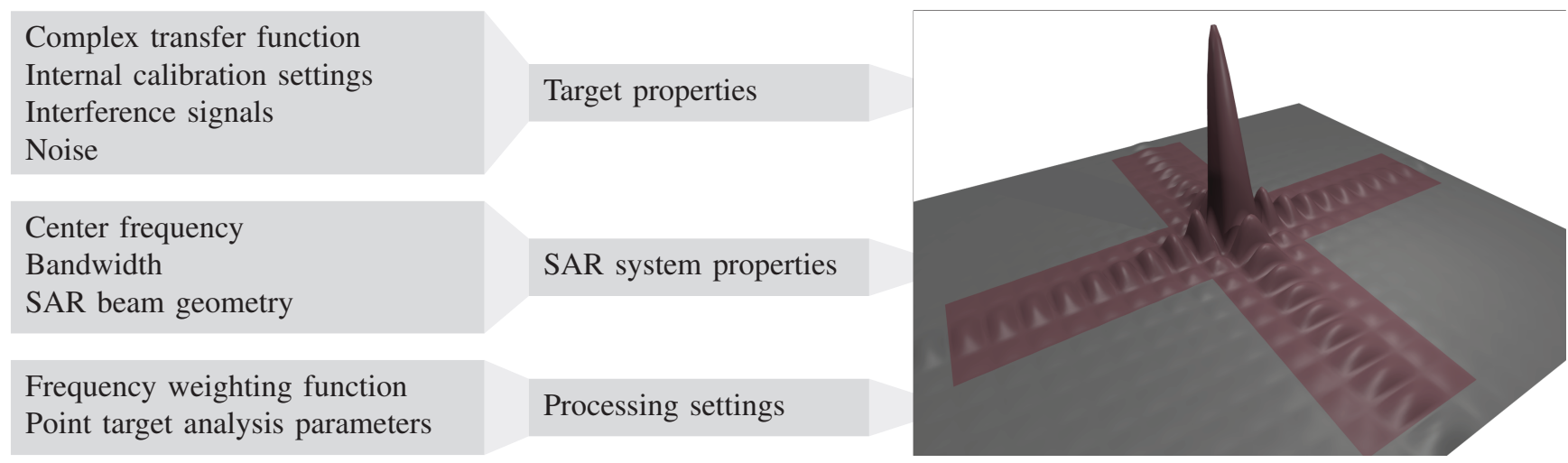

Fig. 1. Overview of influences which affect the point-target response. On the right, an ideal point-target impulse response is shown, for which a possible integration area (cross) for the derivation of the absolute calibration factor $K$ is highlighted.

(e. g. center frequency and bandwidth) and can include updated target properties (due to a possible internal calibration of the transponder).

Such a simulator allows to tie secondary but observable target properties like its (possibly temperature dependent) transfer function to its essential property for absolute radiometric calibration: its radar reflectivity. Furthermore, a frequency dependence is not only introduced by the target itself. The SAR processing is usually frequency dependent as well, and the application of a weighting function during processing for sidelobe suppression is one example. If these processing features are not compensated during external calibration, then the same target might lead to different calibration results depending on the processing parameters. This effect is especially relevant for the absolute cross-calibration of different SAR systems and processors. Therefore, by incorporating a target correction coefficient into the absolute SAR system calibration, a more accurate target reflectivity can be obtained.

The paper is organized as follows. In the next section, relevant aspects of absolute radiometric calibration will be explained. Ideal and non-ideal point targets will be described in Sec. III. A specialized point-target SAR simulator was developed, whose design will be outlined in Sec. IV, and influences of exemplary target deficiencies on the target backscatter, as analyzed by the simulator presented in Sec. IV, will be shown in Sec. V.

\section{Absolute Radiometric CALibration}

The absolute radiometric calibration is the last step in the calibration effort. Usually, only one absolute calibration factor $K$ is derived for every SAR system, i.e. $K$ is independent, for instance, of mode settings or the current instrument temperature. This equalization is achieved by the preceding relative calibration. In contrast to relative calibration, which is partly performed on unfocused SAR data and special internal calibration pulses, $K$ is determined from the final, focused SAR image.

In order to derive the terrain backscatter coefficient of any image pixel in (absolute) units of $\mathrm{dBm}^{2} / \mathrm{m}^{2}$, it is compared with the perceived radar cross section (in $\mathrm{dBm}^{2}$ ) of a known point target. Two methods exist to derive the perceived pointtarget RCS from a SAR image:

- Peak method: Derivation based on the peak pixel value of the impulse response.

- Integral method: Derivation considers the complete impulse response, which extends over several image pixels (see Fig. 1 and [7] for a detailed description).

The integral method is seen to be more robust with respect to a slightly defocused image [7]. Spreading of the point target impulse response might be the result of improper processing (e. g. imperfect platform motion compensation) as well as an imperfect transponder transfer function. It will be shown that the integral method has clear advantages over the peak method especially with respect to imperfect targets.

The term perceived $R C S$, here denoted by $\sigma_{p}$, needs some further explanation. The radar cross section $\sigma$ of any body is defined as the scaled ratio of the scattered to the incident power, as in

$$
\sigma=\lim _{R \rightarrow \infty} 4 \pi R^{2} \frac{\left|\mathbf{E}_{s}\right|^{2}}{\left|\mathbf{E}_{i}\right|^{2}}
$$

where $R$ is the target/observer distance, and $\mathbf{E}_{s}$ and $\mathbf{E}_{i}$ are the scattered and incident electrical fields (see e.g. [8]). In general, the RCS is frequency dependent. Also, the RCS is a scalar, which only depends on amplitudes; the phase response has no influence on the target RCS. Since the target RCS (which is inversely proportional to the absolute calibration factor $K$ to be determined) is only derived after processing by using one of the two above mentioned methods, the pixel brightness or perceived RCS is nevertheless affected by the target phase response. An imperfect (i.e. non-linear) phase response results, for instance, in a broadening of the impulse response while reducing the amplitude of the peak. The RCS of the target according to Eq. (1) would be unaltered, the perceived RCS would be different though. With the proposed point target simulator, differences in the perceived RCS can be quantified.

It has to be noted that background (clutter) and noise compensation as described in [7] is irrelevant for the simulation, as these effects are neglected in the simulation. Also, the discussion about the proper terrain model [9] can be neglected since a flat surface is assumed. 


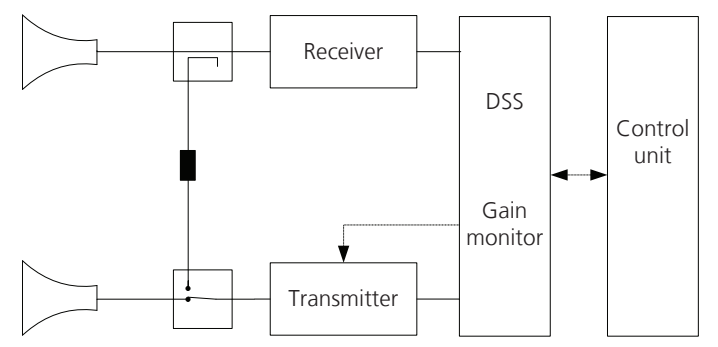

Fig. 2. Functional block diagram of a transponder including internal calibration, from [10]. DSS denotes the digital sub-system, which allows the implementation of a programmable delay line and different internal calibration strategies.

\section{Point TARgets}

\section{A. General Point Targets}

The radar cross section $\sigma$ as defined in Eq. (1) is the most important point-target feature in terms of absolute radiometric calibration. Two types of man-made point targets are commonly used for SAR system calibration: passive corner reflectors and active transponders (Fig. 2). Both types allow a sufficient RCS for a good target-to-clutter separation. Other passive targets like cylinders and spheres have different backscatter properties which render them interesting for some applications, but their geometrical size needs to be larger in comparison to a corner reflector to yield the same RCS.

In the following, the frequency dependence of the RCS of different target types shall be highlighted. The approximate RCS $\sigma_{t}$ (derived from geometrical optics) of a mechanically perfect trihedral corner reflector at perfect alignment is given by [11]

$$
\sigma_{t}=\frac{4 \pi}{3} \frac{a^{4}}{\lambda^{2}}
$$

where $a$ is the inner leg length and $\lambda$ is the wavelength. On the other hand, the RCS $\sigma_{a}$ of an active target (transponder) can be stated as [12]

$$
\sigma_{a}=\frac{\lambda^{2}}{4 \pi} G_{r} G_{e} G_{t}
$$

where $G_{r}$ and $G_{t}$ are the receive/transmit antenna gains and $G_{e}$ is the electronic amplification, respectively. The product $G_{e} G_{r} G_{t}$ is also called the transponder loop gain. The antenna gain in Eq. (3) can be expressed as $4 \pi A_{e} / \lambda^{2}$, where $A_{e}$ is the effective antenna aperture. Therefore, the dependence of RCS on frequency $f$ is identical for both the corner reflector and the transponder. The RCS varies with $f^{2}$ in both cases. In contrast, the maximum RCS of a cylinder is given as [8]

$$
\sigma_{c}=2 \pi \frac{a b^{2}}{\lambda}
$$

where $a$ is the cylinder radius and $b$ its height. The RCS is proportional to $f$, and this dependence also holds for cylinders mounted on a conducting ground plane (tophat reflector) [13]. As a last example, the RCS of a sphere is constant over frequency and is given by [8]

$$
\sigma_{\circ}=\pi a^{2}
$$

where $a$ is the radius of the sphere.

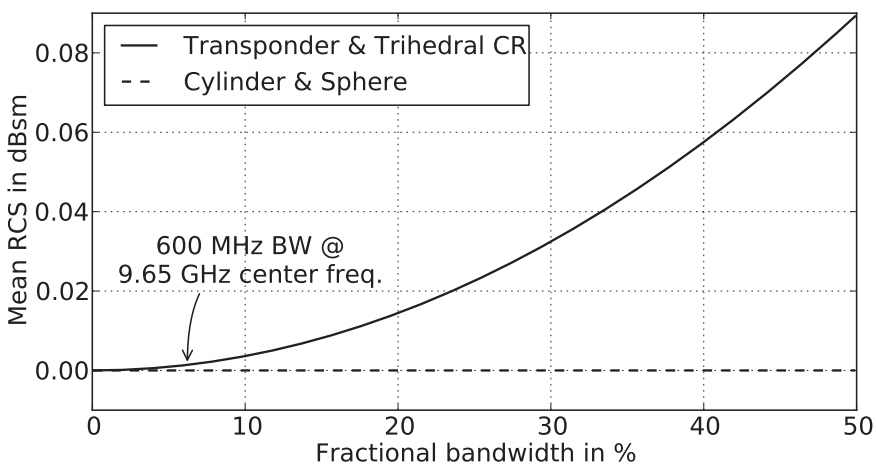

Fig. 3. The average RCS increases for trihedrals and transponders if the bandwidth is increased.

A SAR image is typically acquired by using linear frequency modulated (also called chirp) signals for subsequent range compression. These signals have a certain bandwidth. The image brigthness for such a point target can only be approximately related to the target RCS at the center frequency exactly because the SAR system makes use of a frequency band and not only a single frequency. The range compression during processing behaves like a (usually weighted) frequency averaging filter.

As was shown before, the RCS even of otherwise perfect active and passive targets have a different dependence on frequency $f$. Consequently, the mean RCS over a given frequency band around the SAR center frequency is different even if the RCS is identical at the center frequency. This effect is small for many SAR systems, but it becomes larger for systems with increased fractional bandwidths and is especially important for future wideband SAR systems.

For instance, a fractional bandwidth of about $50 \%$ results in a mean transponder or trihedral RCS which is about $0.1 \mathrm{~dB}$ larger than the mean RCS of a cylinder or sphere despite an identical RCS at the center frequency (also see Fig. 3). For systems with lower fractional bandwidths (e. g. $600 \mathrm{MHz}$ at $9.65 \mathrm{GHz}$, which corresponds to a fractional bandwidth of $6 \%$ ), this effect can be neglected (the difference is $0.001 \mathrm{~dB}$ ).

Therefore, even ideal active and passive point targets cannot be used interchangeably in principal for accurate radiometric SAR calibration. This becomes increasingly important for future wideband SAR systems. Instead, when the calibration factor is stated, the target type and fractional bandwidth used for deriving it should be stated as well. Depending on SAR processing (e. g. the shape of the range weighting filter), other factors might need to be considered. Once this information is provided, a target correction coefficient can be computed to compare calibrated image intensities even if different types of point targets were used. The determination of this target correction coefficient is the aim for the point target SAR simulator, which is going to be described in Sec. IV.

\section{B. Ideal Point Targets}

To conclude the discussion on point targets, an ideal point target shall be defined here as a target which exhibits an RCS independent of frequency (constant amplitude and phase 


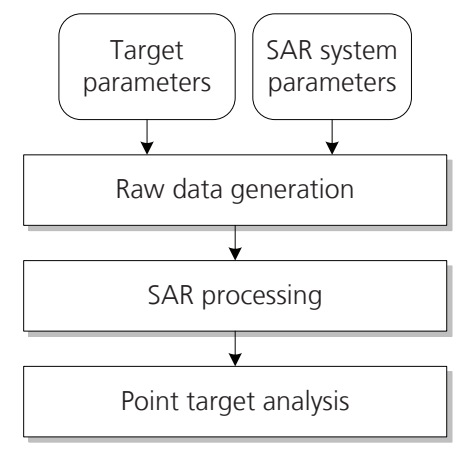

Fig. 4. Principal structure and data flow of the point target SAR simulator.

response). It is convenient to define such a theoretical target since the target correction coefficient is a relative figure which describes the difference between the perceived RCSs of two different targets. This can be noted as

$$
\Delta \sigma_{p}=\sigma_{p}-\sigma_{p, \text { ref }}
$$

where $\sigma_{p}$ represents the perceived RCS of the device under test and $\sigma_{p \text {,ref }}$ serves as the reference. The perceived RCS of an ideal target, as defined above, will be used as the reference in the following point target SAR simulations.

\section{Point TARget SAR Simulator}

The idea of the point target SAR simulator is to model the complete absolute radiometric calibration process: A non-ideal point target is placed within an artificial scene, the raw data as seen by a specific SAR system is simulated, the data is focused, and finally a point target analysis is conducted. By performing a set of simulations with varying target parameters, the relative effect of these parameters on the derived perceived target RCS can be stated.

The program structure, as shown in Fig. 4, is organized as follows:

1) Raw data generation, incorporating reference target deficiencies (e.g. frequency-dependent transfer function) and SAR system specific settings (e.g. chirp settings).

2) SAR data processing, which focuses the generated raw data.

3) Point-target analysis, which extracts point target parameters like its perceived RCS from the focused image.

The simulator does not model all possible influences on the deducted perceived RCS, it focuses solely on deficiencies caused by the point target. For instance, no clutter model is incorporated, and the instrument internal calibration and processing is assumed to be ideal. In this respect, it is contrary to known scientific or commercial SAR simulators, which focus on aspects like mission simulation [14], motion compensation [15] or on statistical features of raw SAR data [16].

The following point target properties are incorporated in the raw data generation step: a set of chained complex transfer functions, noise introduced by the transponder (defined by the signal-to-noise ratio), interference signals (e.g. due to imperfect LO-RF isolation of mixers or multipath effects), and different internal calibration strategies. The raw data is

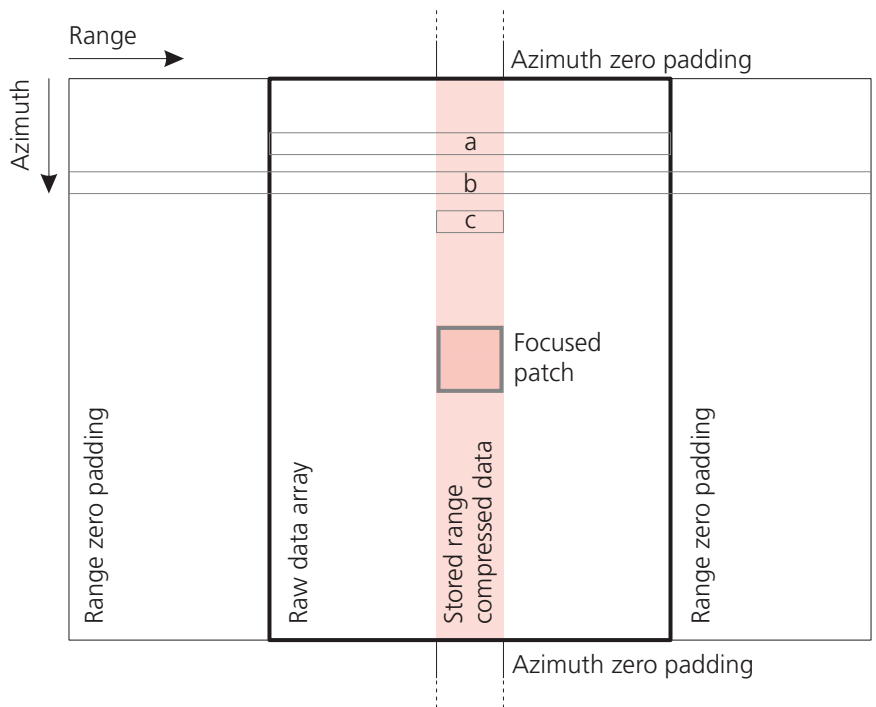

Fig. 5. Data arrays during range and azimuth processing.

generated range line by range line. For reasons of efficiency, a distinction is being made between azimuth time dependent contributions (noise) and constant contributions (transfer functions).

The goal of the SAR data processing is to perform a subsequent point target analysis. As was described in Sec. II, only a few pixels around the point target response are required for the integral method, i.e. it is not necessary to focus the complete scene. This fact can be exploited during processing to significantly reduce memory consumption and processing time. The processing steps can be summarized as follows, where the layout of the data arrays is shown in Fig. 5: First, range and azimuth filters are generated in the frequency domain, which are derived from ideal chirp signals and include weighting (e.g. Hamming window, important since it introduces a frequency dependence). Then, for each generated raw data range line $a$, a conversion from the time to the frequency domain is executed. Zero-padding results in range lines $b$. After multiplication with the range filter and conversion back to the time domain, only the central part $c$ of the range-compressed signal is stored in the intermediate array (highlighted in Fig. 5). Since the raw range lines are generated one by one inside of the processing loop, the main memory requirement is driven by the vertical array size which holds the range compressed data. The algorithm for azimuth compression is identical in principal. Since only the central patch is required for subsequent point target analysis, only a fraction of the total number of azimuth lines actually needs to be processed.

Many processing steps which are found in an operational SAR processor have been deliberately left out. Therefore, range cell migration, instrument antenna pattern compensation, pointing correction, motion compensation, etc. have been excluded. Instead, the raw data is generated in such a way that the mentioned effects do not need to be reversed during processing. This results in a processor which does not introduce any additional perceived RCS deviations not caused 


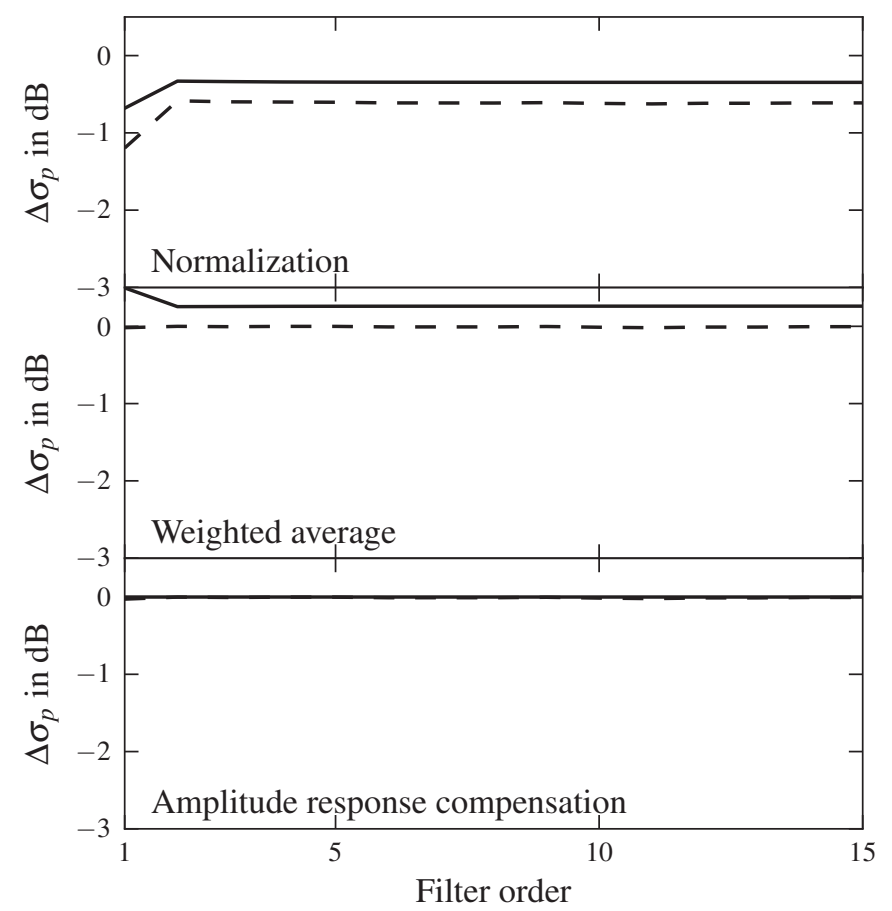

(a) Bessel filters.

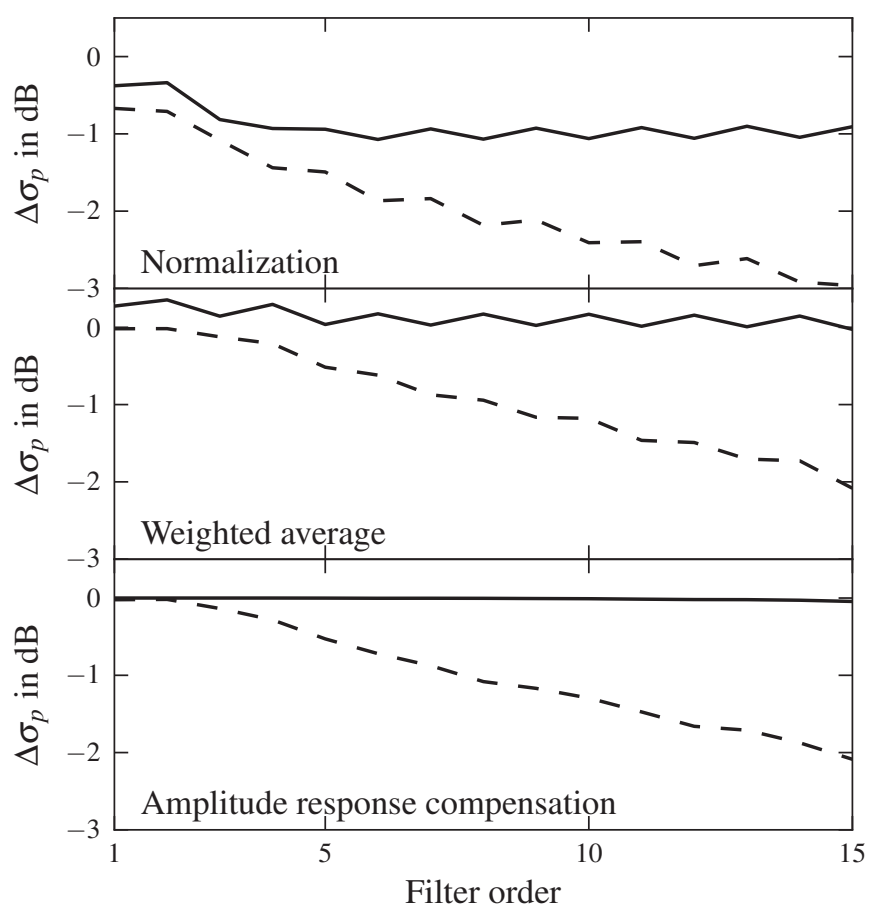

(b) Chebychev filters.

Fig. 6. Simulation results for two filter types and three transponder internal calibration strategies. The deviation of the perceived RCS $\Delta \sigma_{p}$ from an ideal target (flat amplitude and phase response) versus filter order is given for the integral (-) and peak method (- - ), respectively. These deviations represent the reference target correction coefficients (TCCs).

by the target during the raw data generation step. Nevertheless, the frequency dependent focusing (e.g. a Hamming window) is included in the processing step.

\section{Exemplary Point Target Deficiencies}

In the following, the effect of exemplary point target properties on the perceived target RCS will be simulated. The focus will be on transfer functions and internal calibration strategies, interference signals, instrument SAR mode settings, and target noise. According to Fig. 4, target and SAR system parameters need to be known for the simulation. The used SAR system parameters are derived from a TerraSAR-X stripmap beam with the notable difference of a $600 \mathrm{MHz}$ bandwidth range chirp (instead of $150 \mathrm{MHz}$ ) if not otherwise noted. As on TerraSAR-X, the range sampling rate is 2.2 times the range bandwidth, and the pulse length is $57 \mu \mathrm{s}$. The integration cross area according to Fig. 1 is 21 samples long in each direction, and the replica for processing is weighted by a Hamming window.

\section{A. Transfer Functions and Internal Calibration Strategies}

The point target under consideration is a transponder according to Fig. 2. Exemplary transponder transfer functions are based on a series connection of four bandpass filters of varying filter order. The filters are of type Chebychev and Bessel. Out of all transponder loop components the filters where chosen as they contribute considerably to the overall amplitude and phase response. The specific filter types exhibit, on the one hand, a nearly linear group delay and a smooth gain drop-off away from the center frequency (Bessel) and on the other hand a response with comparably large group delays and several amplitude ripples (Chebychev). Different results are therefore to be expected for the point-target analysis after focusing.

The digital sub-system (DSS, see Fig. 2), the unit between the receiver and transmitter chains, allows the implementation of different internal calibration strategies. Internal calibration is here understood as the modification of the transponder transfer function to ensure a stable point-target response even under varying thermal conditions or after component aging. In this example, simulation results for three different strategies will be shown.

1) Strategy 1 ("normalization") adjusts the transponder loop gain according to the gain at one (here: the center) frequency. The actuator can be a variable attenuator (no frequency dependency) in either or both the receiver or transmitter paths.

2) The second strategy ("weighted average") considers the transponder loop gain over the relevant bandwidth and weights it with the frequency dependency of the processor (e.g. Hamming window). The average yields an amplitude correction term, which can be reversed in hardware, again, by a variable attenuator.

3) In the third approach ("amplitude response compensation"), the amplitude frequency response is measured by the digital sub-system, and the amplitude response is corrected in such a way that it becomes flat again. This frequency (and therefore time) dependent approach must be implemented in the DSS. 


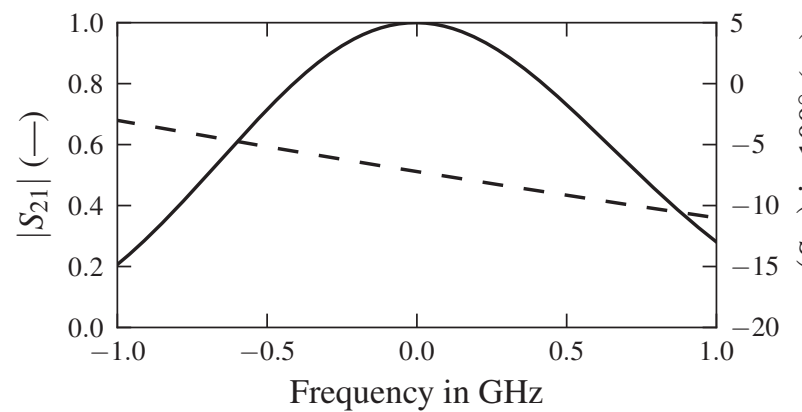

(a) Transfer function.

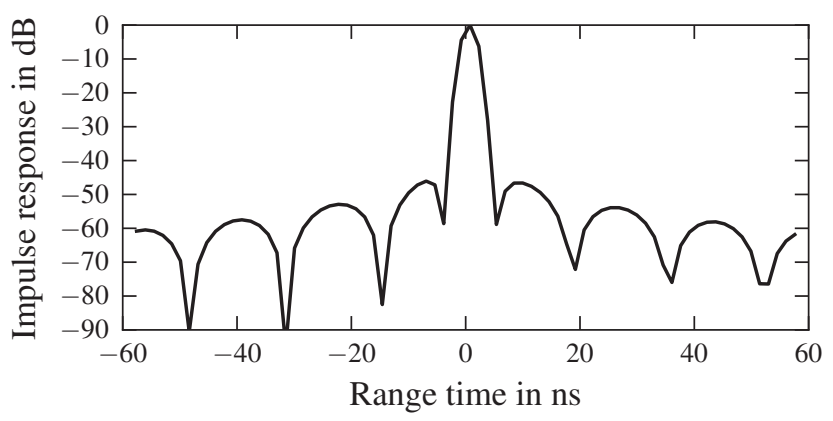

(b) Normalized range cut after focusing (first internal calibration strategy). The shift in range was compensated for this plot so that the peak is located at $0 \mathrm{~ns}$.

Fig. 7. Characteristic plots for a Bessel filter of order 10. Due to the nearly linear phase response, the impulse response looks practically identical to an ideal impulse response.

An ideal fourth approach ("perfect compensation"), in which the internal calibration can completely compensate the amplitude and phase response, results in a target correction factor of zero according to Eq. (6) and is therefore not explicitly pointed out in the resulting plots.

The simulation results are summarized in Fig. 6. The plots show the variation $\Delta \sigma_{p}$ of the perceived target RCS $\sigma_{p}$ for the integral (-) and peak (- - ) methods. In the target design phase, the effect of concrete RF component specifications on the perceived target RCS now becomes apparent. On the other hand, reference target correction coefficients can be stated for observed transfer function variations during operation.

The Bessel filter has a nearly linear phase response, as can be exemplary seen in Fig. 7(a) for a filter order of 10 . In the focused SAR image, this results in a in range shifted impulse response, but the impulse shape is not greatly modified (see Fig. 7(b)). The amplitude response acts like a weighting function and shifts energy away from the peak to the side lobes. Therefore, the peak method underestimates the RCS for the normalization strategy, but achieves a perfect compensation for the weighted average strategy (upper and middle plot in Fig. 6(a)). The amplitude response compensation works very well for a Bessel-style transfer function for both analysis methods (peak and integral) since the phase response can well be neglected (lower plot in Fig. 6(a)).

The amplitude and group delay of the Chebychev filters is much less smooth and linear (Fig. 8(a)). The phase response results in a spreading of the impulse response (Fig. 8(b)),

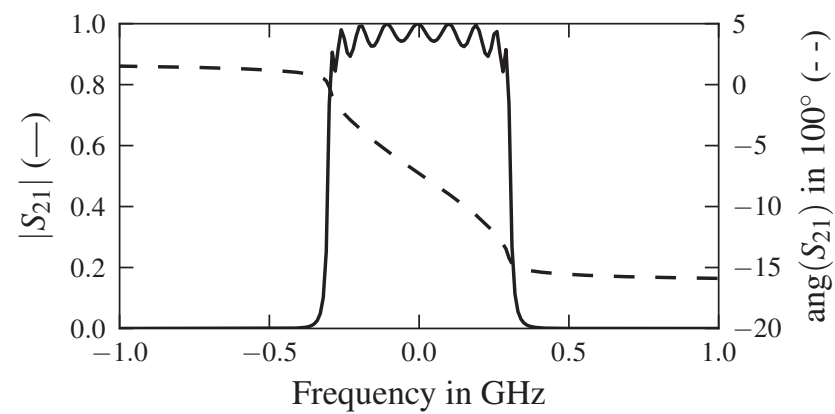

(a) Transfer function.

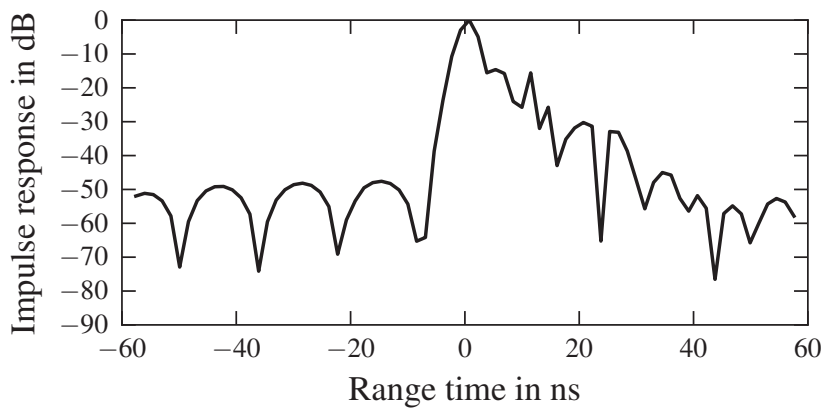

(b) Normalized range cut after focusing (first internal calibration strategy). The shift in range was compensated for this plot so that the peak is located at $0 \mathrm{~ns}$.

Fig. 8. Characteristic plots for a Chebyshev filter of order 10. The imperfect (non-linear) phase response results in a considerable spreading or smearing of the impulse response.

which of course can be better compensated by the integral method for all three internal calibration strategies. If the amplitude response is perfectly compensated as in the third strategy (lower plot in Fig. 6(b)), then the impulse response spreading due to the imperfect phase response is very well neutralized by the integral method.

In summary, a set of realistic transfer functions have been analyzed. Both the amplitude and phase response result in a spreading of the ideal impulse response. The presented internal calibration strategies were focused on compensating the amplitude response function.

In general, independent of the transfer function or the analysis method, a target correction coefficient (TCC) was computed, some reaching $1 \mathrm{~dB}$ even for the preferred integral method. These TCCs can subsequently be applied during the absolute radiometric calibration of the SAR system to improve the radiometric accuracy of image products.

\section{B. Interference Signals}

The output signal of an active target might suffer from signal interference, so that the output signal equals the amplified input signal plus a sum of coherent or non-coherent interference signals. Three types of interference signals shall be distinguished here:

- Continuous wave signals

- Attenuated and delayed versions of the input signal (e.g. caused by coherent multipath effects) 


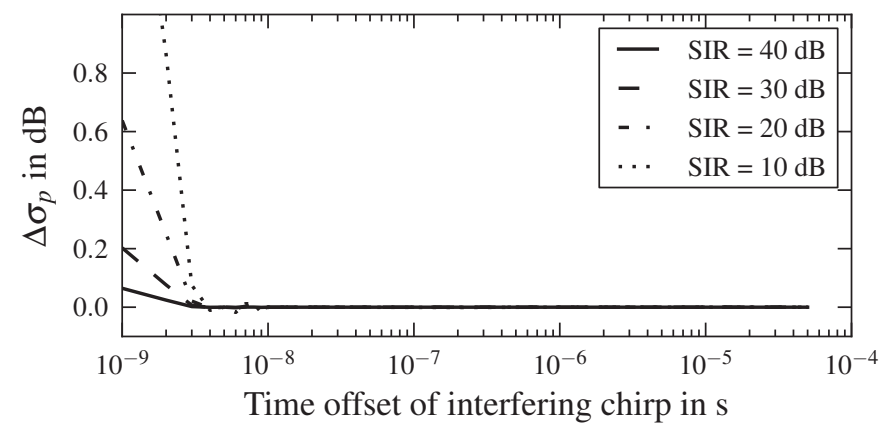

Fig. 9. Effect of coherently interfering chirp signals with a time offset (e.g. due to transmit/receive antenna coupling), integral method. The effect of overlaid chirp signals is only considerable for time offsets below $10 \mathrm{~ns}$.

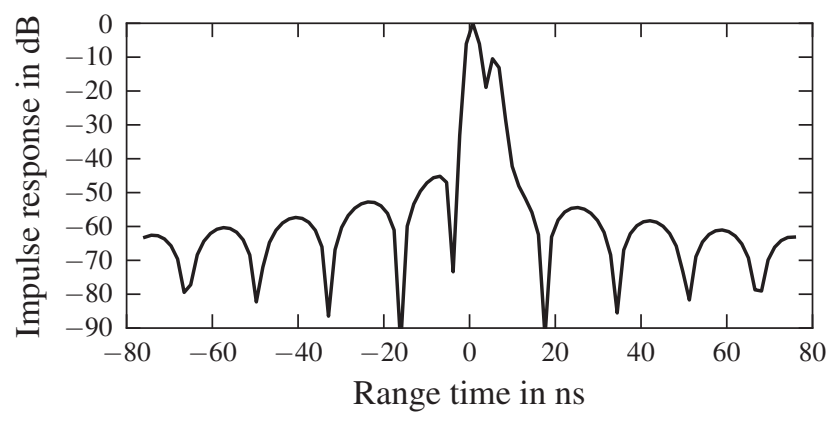

Fig. 10. Exemplary range cut through normalized impulse response for an interfering chirp with SIR $=10 \mathrm{~dB}$ and a time offset of $5 \mathrm{~ns}$. The smaller peak at $5 \mathrm{~ns}$ results in a calibration error.

- Bandwidth-scaled and attenuated versions of the input signal

The amplitude of the interference signals shall be denoted by the signal-to-interference ratio

$$
\operatorname{SIR}=\frac{P_{\text {original signal }}}{P_{\text {interference signal }}}
$$

where $P$ is average power.

A continuous wave signal might stem from an imperfect isolation of the local oscillator signal at a mixer stage or be the result of an EMC problem so that the PC or power supply clock signal penetrates the RF path. These signal contributions are well filtered out by the matched filter during processing. Simulations showed that even an atypically powerful $\mathrm{CW}$ signal with a SIR $=0 \mathrm{~dB}$ results in a $\Delta \sigma_{p}$ of only $0.1 \mathrm{~dB}$. Therefore, in terms of radiometric calibration, $\mathrm{CW}$ interference signals of moderate levels will not influence the derived absolute calibration factor $K$.

The second type of interference, an attenuated and delayed but coherent replica of the input signal, can result from a transmit/receive antenna coupling. This is only relevant if the target allows reception and transmission of a pulse at the same time, i.e. the target pulse delay is less than the pulse length. In the simulation it was assumed that the replica is attenuated with respect to the original signal. This is a practical constraint since otherwise the receiver or transmitter chain would typically be operated in saturation. A repeated feedback was neglected (the pulse delay of the repeated signals would be even larger and the signal further attenuated). The simulation results for a set of SIRs and pulse delays are summarized in Fig. 9. What happens in terms of the point target analysis is that a secondary peak appears in the final image, whose amplitude is attenuated by the SIR and whose range offset is given by the time delay. If the time delay is small, then the secondary peak becomes visually indistinguishable from the main peak. An exemplary range cut is shown in Fig. 10. Since the spurious peak drifts away and out of the integration area for increasing pulse delays, no effect is observed for pulses which are delayed by more than $10 \mathrm{~ns}$ as long as the integration area stays centered around the peak location. The cutoff value of about $10 \mathrm{~ns}$ depends on the chosen size of the integration area for the integral method. For the peak method, the effect of coherently superimposed but delayed replicas is even less strong since only one sample of the side lobe contributes to the error.

The third type of examined interference signals, a bandwidth scaled and attenuated but not delayed version of the replica, can originate from intermodulation products caused by nonlinear RF components. For those signals, the modulation rate of the linearly frequency-modulated signal is changed with respect to the original signal. Simulations showed that these signals behave like non-coherent signals (comparable to the aforementioned $\mathrm{CW}$ signals) and therefore add to the perceived noise floor. Due to the suppression by the matched filter, they do not considerably influence the derived absolute calibration factor.

\section{Instrument SAR Mode Settings}

The simulator's raw data generation step according to Fig. 4 is influenced by target properties as well as by SAR system parameters. In this section, the target parameters shall be held constant and one parameter of the SAR mode ("beam") settings shall be modified. Here, the range bandwidth is varied from $5 \mathrm{MHz}$ to $600 \mathrm{MHz}$, emulating the transition from wide swath/low resolution to spotlight/high resolution SAR modes. Two targets shall be simulated, once again modeled by chaining four filters, 10th order, of two filter types, respectively.

The results of the simulations for the preferred integral method are shown in Fig. 11. All internal calibration strategies work well for both transponders if the bandwidth is small since the transfer function is nearly constant. For larger bandwidths, the relative performance of different internal calibration strategies can be observed. The amplitude response compensation results in a perfect compensation for both analyzed transponders over all bandwidths. The simplest internal calibration strategy, normalization, performs worst, so that the perceived RCS of the Chebychev-filter-based transponder is more than $1 \mathrm{~dB}$ larger for a low-resolution $(5 \mathrm{MHz}) \mathrm{SAR}$ mode than for a high-resolution $(600 \mathrm{MHz})$ mode.

To compensate for these effects, the transponder can either be calibrated separately for each expected SAR mode setting prior to the external calibration of the SAR system, or the differences of up to $1 \mathrm{~dB}$ can be determined analytically by the presented simulator. 


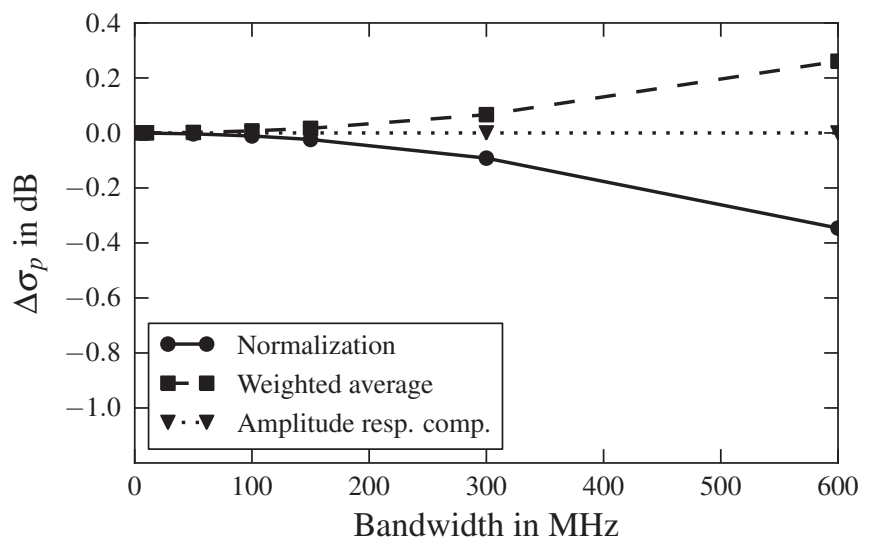

(a) First transponder based on 10th order Bessel filters.

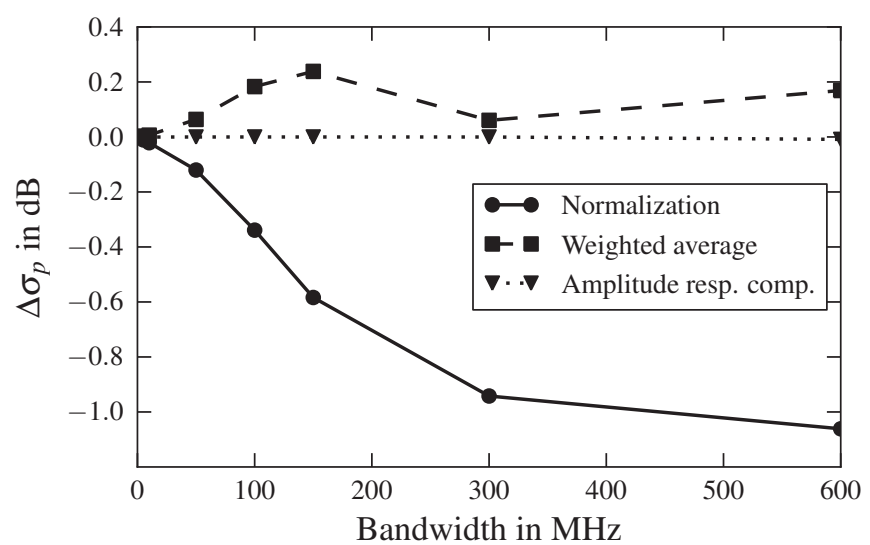

(b) Second transponder based on 10th order Chebychev filters.

Fig. 11. Simulation results (integral method) for two transponders which are designed for a range bandwidth of $600 \mathrm{MHz}$ (transfer functions modeled by different filter types). If the transponder is operated for different SAR modes with varying range bandwidths, a deviation of the perceived RCS $\sigma_{p}$ can be observed depending on the utilized internal calibration strategy.

\section{Noise}

An active point target will always add noise to the received SAR signal before retransmission. Sources of this noise include thermal and shot noise in several RF components, and possibly digitization noise if a digital sub-system is used. In the point target SAR simulator, additive white Gaussian noise is assumed (constant power density and normal amplitude distribution), which can be parameterized by the signal-tonoise ratio. In the simulator, a random number generator is used which generates a separate noise signal for each range line.

Since noise is an uncorrelated signal, it gets considerably suppressed during impulse compression in the processing step. The noise power still exists in the focused image, but it stays spread equally over all image pixels, while the coherent point target response gets focused to a peak. The noise suppression in the power image equals the processing gain, which is given by the product of the pulse bandwidth and the pulse length [11]. For the exemplary signal considered in this section, a noise suppression of $45 \mathrm{~dB}$ results, so that even a signal-to-noise ratio of e.g. only $10 \mathrm{~dB}$ in the active target would still be sufficiently low. This result can be confirmed numerically by the point target SAR simulator.

It can be concluded that the noise figure of an active target is usually not critical due to the noise suppression during processing.

\section{CONCLUSION}

In this paper, a closer look at the properties of imperfect or real point targets was given. It was found that the radar cross section is not enough to characterize a point target as a calibration normal for accurate radiometric SAR calibration. Instead the perceived RCS of those targets needs to be considered, which takes the processing and point target analysis into consideration.

Based on this consideration, a point target SAR simulator was developed. It allows the end-to-end simulation of the absolute radiometric calibration process when imperfect targets are used. The simulator can compute target correction coefficients (TCCs) for each target and SAR mode, which indicate the differences in perceived RCS with respect to an ideal target. The properties which can be analyzed by the implemented SAR simulator are the target transfer function, different target internal calibration strategies, interference signals, instrument SAR mode settings, and noise. Exemplary results for each imperfection type were presented, which can be helpful in developing a new target design.

Using a dedicated point target SAR simulator during the absolute radiometric calibration, more accurate absolute radiometric calibrations become feasible by reducing the remaining point target uncertainties. In the presented quantitative examples, target correction coefficients larger than $1 \mathrm{~dB}$ were found. Target and mode dependent point target correction coefficients should therefore be incorporated into the derivation of the absolute calibration factor $K$ for accurate SAR system calibration.

\section{ACKNOWLEDGMENT}

The authors would like to thank Andreas Danklmayer, DLR, for constructive criticism and valuable discussions.

\section{REFERENCES}

[1] A. Freeman, "SAR calibration: An overview," IEEE Transactions on Geoscience and Remote Sensing, vol. 30, no. 6, pp. 1107-1121, Nov. 1992.

[2] M. Schwerdt, B. Bräutigam, M. Bachmann, B. Döring, D. Schrank, and J. Hueso Gonzalez, "Final TerraSAR-X calibration results based on novel efficient methods," IEEE Transactions on Geoscience and Remote Sensing, vol. 48, no. 2, pp. 677-689, Feb. 2010.

[3] M. Shimada, M. Watanabe, A. Rosenqvist, and T. Tadono, "PALSAR calibration and validation - 2005 update," International Geoscience and Remote Sensing Symposium, pp. 3365-3368, Jul. 2005.

[4] A. Luscombe, "Image quality and calibration of RADARSAT-2," IEEE International Geoscience and Remote Sensing Symposium, pp. II-757II-760, Jul. 2009.

[5] J. Johnson, L. Williams, E. Bracalente, F. Beck, and W. Grantham, "SEASAT - A satellite scatterometer instrument evaluation," IEEE Journal of Oceanic Engineering, vol. 5, no. 2, pp. 138-144, Apr. 1980.

[6] E. Attema, M. Davidson, P. Snoeij, B. Rommen, and N. Floury, "Sentinel-1 mission overview," IEEE International Geoscience and Remote Sensing Symposium, pp. I-36-I-39, Jul. 2009. 
[7] A. L. Gray, P. W. Vachon, C. E. Livingstone, and T. I. Lukowski, "Synthetic aperture radar calibration using reference reflectors," IEEE Transactions on Geoscience and Remote Sensing, vol. 28, no. 3, pp. 374-383, May 1990.

[8] E. F. Knott, J. F. Schaeffer, and M. T. Tuley, Radar cross section. Its prediction, measurement and reduction. Dedham, MA: Artech House, 1985.

[9] D. Small, N. Miranda, and E. Meier, "A revised radiometric normalisation standard for SAR," IEEE International Geoscience and Remote Sensing Symposium, pp. IV-566-IV-569, Jul. 2009.

[10] B. J. Döring, P. Looser, M. Jirousek, M. Schwerdt, and M. Peichl, "Highly accurate calibration target for multiple mode SAR systems," European Conference on Synthetic Aperture Radar, vol. 8, Jun. 2010.

[11] H. Klausing and W. Holpp, Radar mit realer und synthetischer Apertur: Konzeption und Realisierung. München: Oldenbourg Wissenschaftsverlag $\mathrm{GmbH}, 2000$.

[12] D. R. Brunfeldt and F. T. Ulaby, "Active reflector for radar calibration," IEEE Transactions on Geoscience and Remote Sensing, vol. GE-22, no. 2, pp. 165-169, Mar. 1984.

[13] J. Silverstein and R. Bender, "Measurements and predictions of the RCS of bruderhedrals at millimeter wavelengths," IEEE Transactions on Antennas and Propagation, no. 7, pp. 1071-1079, Jul. 1997.

[14] R. Speck, "SETES - SAR end-to-end simulator." [Online]. Available: http://http://www.dlr.de/hr/en/desktopdefault.aspx/tabid-2434/ 3770_read-5675/

[15] Array Systems Computing Inc., "Synthetic aperture radar simulation toolbox." [Online]. Available: http://www.array.ca/applications/radar/ sar $\backslash$ toolbox.php

[16] G. Franceschetti, M. Migliaccio, D. Riccio, and G. Schirinzi, "SARAS A synthetic aperture radar (SAR) raw signal simulator," IEEE Transactions on Geoscience and Remote Sensing, no. 1, pp. 110-123, 1992.

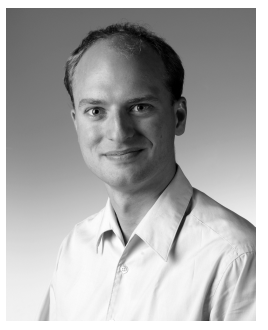

Björn J. Döring Björn Döring received the Dipl.Ing. degree in electrical engineering from the Technical University Berlin, Germany, in 2005. Since 2006, he has been with the Microwaves and Radar Institute, German Aerospace Center (DLR), Wessling, Germany. His research concentrates on satellite SAR calibration with a focus on absolute radiometric calibration. His design and characterization of passive and active calibration targets contribute to precisely calibrated SAR systems like TerraSAR-X, TanDEM-X, and GMES Sentinel-1.

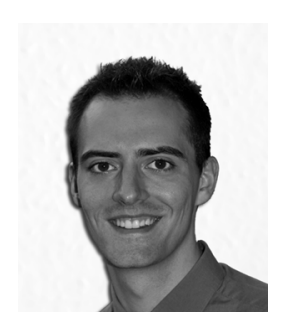

Philipp Looser Philipp R. Looser received the B. Sc. degree in electrical power systems and the M. Sc. degree in micro- and optoelectronics from the Swiss Federal Institute of Technology (ETH), Zurich, Switzerland. Since 2010 he has been with the Microwaves and Radar Institute, German Aerospace Center (DLR), Wessling, Germany. His work focuses on the development of new transponders for spaceborne SAR system calibration.

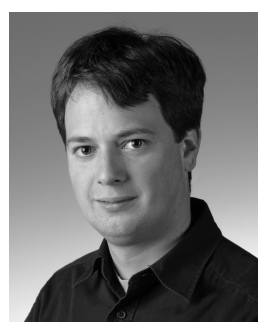

Matthias Jirousek Matthias Jirousek received the Dipl.-Ing. degree in electrical engineering from the Karlsruher Institute of Technology, Karlsruhe, Germany, in 2002. Since 2003, he has been with the Microwaves and Radar Institute, German Aerospace Center (DLR), Wessling, Germany, where he is working on microwave sensor system development. $\mathrm{He}$ is responsible for the research on a passive microwave imaging experimental spectrometer system based on aperture synthesis. His current research concentrates on the highly digitized wideband radar instrument Gigarad and the development of precise and flexible active calibration targets for future satellite SAR calibration missions with a focus on absolute radiometric calibration.

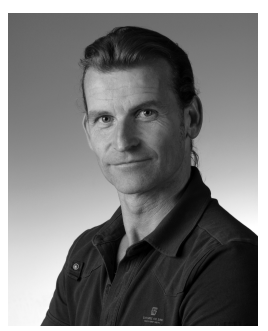

Dr.-Ing. Marco Schwerdt Marco Schwerdt received the Dipl.-Ing degree in electrical engineering and the Dr.-Ing. degree, with a thesis on electrooptical E-field sensors, from the Technical University Berlin, Germany. Since 1998, he has been with the Microwave and Radar Institute, German Aerospace Center (DLR), Wessling, Germany, where he is working on SAR calibration methods and performance analysis tools. Since 2000, he has been the head of the Radar Calibration Group performing various radar calibration activities for different SAR missions like XSAR/SRTM or the ScanSAR mode of ASAR/Envisat. He was responsible for the successful calibration of the German TerraSAR-X and TanDEM-X satellites, launched in 2007 and 2010 respectively. Furthermore, as part of the Global Monitoring Environment and Security Program (GMES), he is responsible for developing the overall SAR system calibration and validation plan for ESA's Sentinel-1 mission. Under his leadership, the DLR's comprehensive radar calibration facilities, including novel tools for product quality control and performance analysis, have been maintained and extended. His major research interest includes the development of innovative and efficient calibration methods.

Dr. Schwerdt received the Der Deutsche Gründerfonds award in 1997 for establishing an enterprise of manufacturing electrooptical field sensors under the patronage of the German Federal Minister for Science and Research. 